Review Article

\title{
New Biologics for the Treatment of Atopic Dermatitis: Analysis of Efficacy, Safety, and Paradoxical Atopic Dermatitis Acceleration
}

\author{
Hong-jiao Qi and Lin-Feng $\mathrm{Li}$ (D) \\ Department of Dermatology, Beijing Friendship Hospital, Capital Medical University, Beijing, China \\ Correspondence should be addressed to Lin-Feng Li; zoonli@sina.com
}

Received 12 January 2021; Accepted 21 May 2021; Published 30 May 2021

Academic Editor: Giuseppe Valacchi

Copyright (c) 2021 Hong-jiao Qi and Lin-Feng Li. This is an open access article distributed under the Creative Commons Attribution License, which permits unrestricted use, distribution, and reproduction in any medium, provided the original work is properly cited.

\begin{abstract}
Atopic dermatitis $(\mathrm{AD})$ is a chronic, inflammatory skin disease with an eczematous rash and itching. Due to undesired adverse effects of traditional systemic treatment, there is still an unmet need for safe and effective long-term therapy for refractory AD. As our understanding of the pathogenesis underlying $\mathrm{AD}$ grows, novel treatments targeting specific molecules have been developed. Here, we discuss the efficacy and safety profiles of these drugs in recent clinical trials. Among their adverse effects, of particular note is $\mathrm{AD}$ acceleration. Although there is still debate about whether certain adverse reactions can be said to be paradoxical adverse events (PAEs), a wide range of PAEs have been reported during biological treatment for chronic immunemediated diseases. Close surveillance of novel biologics is crucial to detect new undescribed paradoxical reactions and to shed light on the convoluted pathogenesis of $\mathrm{AD}$.
\end{abstract}

\section{Introduction}

Atopic dermatitis $(\mathrm{AD})$ is one of the most common chronic, inflammatory, relapsing skin diseases [1]. Up to $17.1 \%$ of adults and $22.6 \%$ of children are diagnosed with $\mathrm{AD}$ each year [2]. AD is a very distressing disease that is characterized by pruritus and dry skin [3]. For patients with moderate-tosevere $\mathrm{AD}$, systematic treatments are often necessary [4]. The use of traditional systemic treatments (systemic corticosteroids, phototherapy, and immunosuppressants) is limited by safety risks and variable therapeutic benefits [5]. Thus, new systemic therapies have been developed recently.

For approximately 20 years, biological agents (BAs) have been widely used in various autoinflammatory and immune diseases [6]. As new emerging drugs come to market, a tradeoff between efficacy and safety is achieved [7]. Dupilumab, an IL-4 and IL-13 inhibitor, was the first biological drug approved by the FDA for the treatment of AD in adults [8]. It is noteworthy for its acceptable low side effect profile (lower rate of conjunctivitis, injection-site reactions, and infections) and high efficacy (36\%-44\% of patients achieve clear or almost clear skin) [9-11]. Other new biologics that selectively inhibit cytokines involved in the inflammatory component of $\mathrm{AD}$ are discussed in our review.

Among the emergent treatment adverse effects, of particular note is $\mathrm{AD}$ acceleration. Although there is still a debate about whether certain adverse reactions can be said to be paradoxical adverse events (PAEs), a wide range of PAEs have been reported during biological treatment for chronic immunemediated diseases [12]. PAEs are defined as the occurrence of a pathological condition that usually responds to this class of drug during biological agent therapy [13].

Insufficient data are available concerning the incidence of PAEs [13]. Most paradoxical reactions have been reported to be connected with anti-TNF therapy; however, it is possible that the number of cases will increase as the number of newly introduced biological agents increases [14]. Representative examples of PAEs are palmoplantar pustular reactions, psoriasiform reactions, and hidradenitis suppurativa (HS) in patients under treatment for rheumatoid arthritis (RA) or inflammatory bowel disease (IBD) [15]. A few reviews and case reports have described PAEs: de novo psoriasis in atopic dermatitis patients treated with dupilumab [16-23], paradoxical head and neck erythema in patients with atopic 
dermatitis treated with dupilumab [24-26], mepolizumabinduced alopecia in severe eosinophilic asthma [27], and secukinumab-induced exacerbation of previously diagnosed psoriasis [15]. Regarding atopic dermatitis acceleration, two cases reported the exacerbation of atopic dermatitis symptoms by ustekinumab in psoriatic patients [28].

This review discusses the efficacy, safety, and possible PAEs of novel biological therapies currently in phase II and phase III clinical trials for moderate-to-severe AD.

\section{Pathogenesis}

Atopic dermatitis is characterized by $\mathrm{T}$ cell-mediated skin inflammation and an impaired skin barrier. The acute phase of $\mathrm{AD}$ is characterized by a strong modulation of Th2 and Th22 immune responses, along with effects on the Th17/IL17 and IL-23 pathways [29, 30]. Barrier-disrupted keratinocytes are potent producers of immunoregulatory cytokines such as thymic stromal lymphopoietin (TSLP), IL-25, and IL-33 [31]. TSLP plays a critical role in activating the Th2 cascade [29]. TSLP and IL-25 activate dendritic cells (DCs) to express OX40 L. OX40 L/OX40 initiates type 2 immune differentiation of T cells. TSLP also induces IL-23 production by human DCs [32]. IL-33 can positively regulate the TSLPdendritic cell-OX40 $\mathrm{L}$ axis, participating in the induction and maintenance of the Th2 response [33, 34]. The Th22 pathway is consistently activated by the Th2 pathway in $\mathrm{AD}$, and both are considered key immune drivers of $\mathrm{AD}[35,36]$. While acute $\mathrm{AD}$ pathogenesis is polarized towards Th2 and Th22 immune responses, chronic $\mathrm{AD}$ lesions additionally exhibit a substantial Th1 component. Th2 cells release IL-4, IL-13, IL-31, and IL-5 [31]. IL-4 and IL-13 disrupt barrier function by downregulating filaggrin (FLG) expression. Furthermore, IL-4 and IL-13 prompt inflammation through the stimulation of IgE production from plasma cells and B cell and plasma cell differentiation [37]. IL-4 and IL-13 also amplify IL-31-induced and histamine-induced pruritus [31]. IL-4 and IL-13 augment the production of CCL17, CCL22, and CCL26. These chemokines, along with IL-5, recruit Th2 cells and eosinophils $[31,38]$. IL-31 stimulates sensory nerves and induces pruritus [39], the itching evokes scratching, and the itch-scratch cycle aggravates barrier disruption [31].

IL-22, the leading Th22 cytokine, was suggested to have a major pathogenic role in epidermal pathology, induce keratinocyte proliferation, and downregulate FLG expression, resulting in barrier dysfunction and epidermal hyperplasia [40]. Th17/IL-17 and IL-23 pathway-associated cytokines (IL-17 and IL-12/23p40) are increased in several AD subtypes, including intrinsic [41], Asian [42], and paediatric $\mathrm{AD}$ [43]. IL-23 is composed of a p19 subunit in addition to a p40 subunit, which is also a component of IL-12. IL-23 also has one receptor subunit in common with IL-12 and IL$12 \mathrm{R} \beta 1$. IL-23 is a crucial player in the expansion and survival of Th17 T cells [44]. IL-17A and IL-17F secreted by Th17 cells can promote eosinophil production [45]. IL-12 secreted by eosinophils, dermal dendritic cells (DDCs), and inflammatory epidermal dendritic cells (IDECs) [45] induces the production of IFN- $\gamma$ [46], which results in the Th2 acute phase-to-Th1 chronic phase switch in AD [45] (Figure 1).

\section{Efficacy, Safety, and Treatment-Emergent AD Adverse Events}

3.1. Targeted to $I L-33$. Etokimab (ANB020) is a humanized anti-human IL-33 monoclonal antibody [47]. A phase 2a clinical trial enrolled 12 moderate-to-severe adult refractory atopic dermatitis patients. A single intravenous dose of placebo (day 7) followed by a single dose of $300 \mathrm{mg}$ intravenous etokimab (day 1) was administered. Eighty-three percent of patients achieved the primary endpoint Eczema Area and Severity Index (EASI) 50, and 33\% achieved EASI 75 at day 29. All patients achieved an EASI 50 response on or before day 57. EASI responses were consistent with the improvement of 5D (5-domain) itch scores (5-D pruritus), SCORing Atopic Dermatitis (SCORAD), Dermatology Life Quality Index (DLQI scores), and Investigator Global Assessment (IGA) score 0/1 achievement [47.48]. Etokimab was well tolerated. Most AEs were mild [47]. The most frequent adverse event was dizziness in $17 \%$ of the placebo arm versus headache in $25 \%$ of the etokimab arm [48].

In a phase $2 \mathrm{~b}$ randomized, double-blinded, placebocontrolled, multidose study (ATLAS trial), 300 adult moderate-to-severe atopic dermatitis patients were treated with etokimab (ANB020) for 16 weeks (ClinicalTrials.gov Identifier: NCT03533751). Each of the etokimab dosing arms failed to meet the primary endpoint (percent change in EASI from baseline to week 16) (GLOBE NEWSWIRE (https://ir.anaptysbio.com/news-releases/news-release-details/ anaptysbio-reports-etokimab-atlas-phase-2b-clinical-trial/) Nov. 08, 2019).

3.2. Targeted to OX40. GBR 830 is a humanized mAb (MAB) against OX40, a costimulatory receptor on activated $\mathrm{T}$ cells [49]. A phase 2a, randomized, double-blind, placebocontrolled study (NCT02683928) evaluated the safety and biological activity of GBR 830 in adults with moderateto-severe AD. Sixty-four eligible adult subjects were randomized $3: 1$ to receive $10 \mathrm{mg} / \mathrm{kg}$ intravenous GBR 830 or placebo on day 1 and day 29 [49]. Primary endpoints included treatment-emergent adverse events (TEAEs) and changes in epidermal hyperplasia and gene expression of biomarkers in lesional skin biopsies at days 29 and 71 [49]. GBR 830 was well tolerated, with equal TEAE distribution (GBR 830, $63.0 \%$ [29/46]; placebo, 63.0\% [10/16]). The most frequent TEAEs in the GBR 830 group than in the placebo group were $\mathrm{AD}$, postprocedural infection, and myalgia [49].

Significant reductions in Th1 (IFN- $\gamma /$ CXCL10), Th2 (IL31/CCL11/CCL17), and Th17/Th22 (IL-23p19/IL-8/S100As) mRNA expression in lesional skin were induced by GBR 830 , but the key cytokines Th2 (IL-4 and IL-13) and Th17/Th22 (IL-17A and IL-22) were not significantly reduced with GBR 830 compared with placebo. Hyperplasia measures (thickness/keratin 16/Ki67) were significantly reduced with GBR $830(P<0.001)$ [49].

Clinical efficacy was the secondary endpoint. At day 71, the proportion of eligible subjects achieving $50 \%$ or greater improvement in EASI score was greater with GBR 830 (76.9\% [20/26]) versus placebo (37.5\% [3/8]). IGA response (IGA score of 0 or 1 ) was achieved by $23.1 \%$ of GBR 830 - 


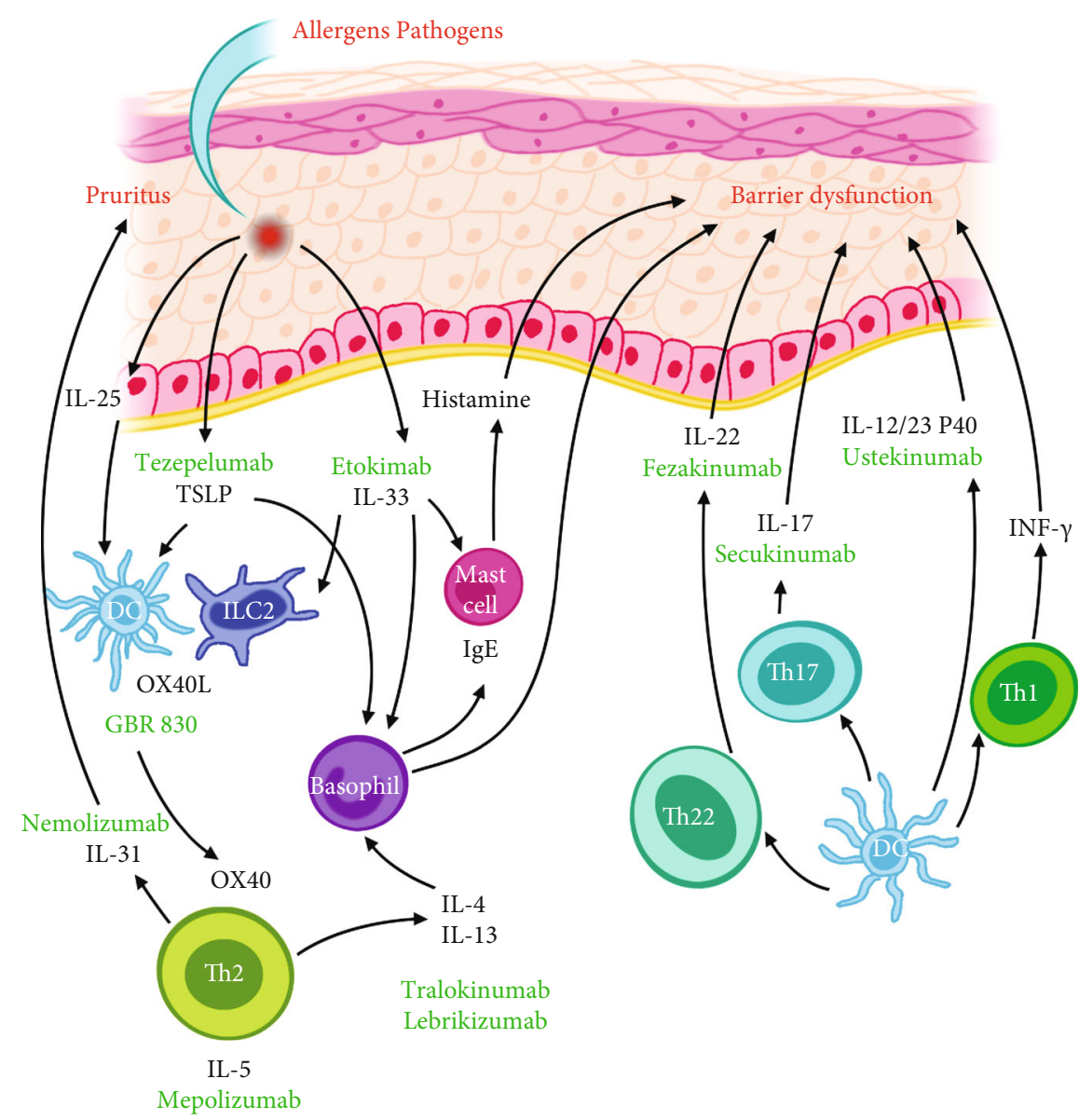

FIGURE 1: The pathogenesis and new corresponding therapeutic biologics in atopic dermatitis.

treated subjects compared to $12.5 \%$ of placebo-treated subjects at day 71. However, SCORAD, body surface area (BSA), and pruritus Numerical Rating Scale (NRS) scores only showed small numerical improvements [49].

\subsection{Targeted to $I L-13$}

3.3.1. Tralokinumab. Tralokinumab is a fully human monoclonal antibody that potently binds to and neutralizes the effects of IL-13 [50]. In a phase 2b study (NCT02347176), 204 adults were randomized $1: 1: 1: 1$ to receive 45,150 , and $300 \mathrm{mg}$ of subcutaneous tralokinumab or placebo every 2 weeks for 12 weeks with concomitant topical glucocorticoids. At week 12, the adjusted mean difference from baseline in EASI score (primary endpoint) was significantly different than that in the placebo group, $150 \mathrm{mg}$ group $-4.36(P=0.03)$ and $300 \mathrm{mg}$ group $-4.94(P=0.01)$, while there was no significant difference in the percentage of participants with an IGA response (coprimary endpoint) at week $12(23.0 \%$ vs. $11.8 \%, P=0.10)$. More responses were found in participants with greater concentrations of biomarkers (DPP-4 and periostin) [50]. Tralokinumab has an acceptable safety profile with TEAEs relative to placebo $(60.8 \%)$ and pooled tralokinumab (66\%). The most common adverse events were upper respiratory tract infections and headache [50].
In two 52-week, randomized, double-blind, placebocontrolled, phase III trials, ECZTRA 1 and ECZTRA 2 (NCT03131648, NCT03160885), adult patients with moderate-to-severe $\mathrm{AD}$ were randomized $(3: 1)$ to receive subcutaneous tralokinumab $300 \mathrm{mg}$ (600 mg loading dose on day 0) or placebo every 2 weeks (Q2W) for 16 weeks. A total of 802 subjects were enrolled in ECZTRA 1, and 794 subjects were enrolled in ECZTRA 2 [51]. Primary endpoints were IGA score of 0 or 1 and EASI 75 at week 16. Patients achieving an IGA score of $0 / 1$ and/or EASI 75 with tralokinumab at week 16 were rerandomized to tralokinumab $300 \mathrm{mg}$ Q2W, $300 \mathrm{mg}$ Q4W, or placebo for 36 weeks [51]. At week 16 , in both trials, the tralokinumab group had a greater proportion of subjects who achieved an IGA score of $0 / 1$ and EASI 75 than placebo. More patients who received tralokinumab vs. placebo achieved an IGA score of 0/1: $15.8 \%$ vs. $7.1 \%$ in ECZTRA $1(P=0.002)$ and $22.2 \%$ vs. $10.9 \%$ in ECZTRA 2 $(P<0.001)$ and EASI 75: $25.0 \%$ vs. $12.7 \%(P<0.001)$ and $33.2 \%$ vs. $11.4 \%(P<0.001)$. More than $50 \%$ of patients who achieved IGA $0 / 1$ at week 16 with tralokinumab Q2W maintained that response to week 52 [51]. Tralokinumab has an acceptable safety profile, with a comparable incidence of AEs between tralokinumab and placebo in the initial treatment period of both studies. Among the most often reported AEs, upper respiratory tract infection and conjunctivitis occurred more frequently with tralokinumab than placebo, 
and atopic dermatitis and skin infection appeared more frequently with placebo [51].

In a phase III, double-blind, randomized, placebocontrolled 32-week trial (ECZTRA 3 NCT03363854), a total of 380 patients were randomized 2: 1 to receive subcutaneous tralokinumab $300 \mathrm{mg}$ or placebo Q2W with TCS for 16 weeks. Patients who achieved an IGA score of $0 / 1$ and/or EASI 75 at week 16 with tralokinumab were rerandomized 1:1 to tralokinumab Q2W or Q4Wfor another 16 weeks [52]. At week 16, more patients treated with tralokinumab than placebo achieved IGA $0 / 1: 38.9 \%$ vs. $26.2 \%(P=0.015)$ and EASI $75: 56.0 \%$ vs. $35.7 \%(P<0.001)$. Of the patients who achieved IGA $0 / 1$ and/or EASI 75 at week 16 with tralokinumab, 89.6\% and 92.5\% treated with Q2W and 77.6\% and $90.8 \%$ treated with Q4W maintained a response at week 32, respectively. At week 32, among patients who did not achieve an IGA $0 / 1$ and EASI 75 with tralokinumab Q2W at 16 weeks, $30.5 \%$ and $55.8 \%$ achieved these endpoints, respectively [52]. The overall rate of adverse events (AEs) was comparable between treatment groups. Viral upper respiratory tract infections, conjunctivitis, headache, upper respiratory tract infections, and injection-site reactions were reported more frequently for tralokinumab than placebo among the most frequently reported AEs [52].

3.3.2. Lebrikizumab. Lebrikizumab is a novel high-affinity monoclonal antibody that binds to soluble IL-13, preventing IL-13R $\alpha 1 /$ IL- $4 \mathrm{R} \alpha$ heterodimerization and inhibiting subsequent signalling [53].

In a phase 2 (NCT02340234) randomized, placebo-controlled, double-blind, phase II study, a total of 209 adult patients with moderate-to-severe $\mathrm{AD}$ were randomized $(1: 1: 1: 1)$ to receive a subcutaneous injection of lebrikizumab $125 \mathrm{mg}$ single dose (SD), $250 \mathrm{mg}$ SD, $125 \mathrm{mg}$ Q4W, or placebo Q4W for 12 weeks after a 2-week TCS run-in [54]. At week 12, significantly more patients achieved EASI 50 (primary endpoint) with lebrikizumab $125 \mathrm{mg}$ Q4W (82.4\%; $P=0.026)$ versus placebo $(62.3 \%)$; patients receiving lebrikizumab SD showed no statistically significant improvements in EASI 50 versus placebo. The percentage of patients who achieved IGA $0 / 1$ at week 12 did not show statistical significance in any lebrikizumab group compared with the placebo group [54]. Lebrikizumab was generally well tolerated. The incidence of AEs was similar between groups (66.7\% all lebrikizumab vs. $66.0 \%$ placebo), mostly mild or moderate. Of the AEs of interest, conjunctivitis (9.6\%), herpetic infections (3.8\%), and eosinophilia without clinical symptoms (3.2\%) occurred more frequently in lebrikizumab-treated patients [54].

In a phase $2 \mathrm{~b}$, double-blind, placebo-controlled, randomized clinical trial (NCT03443024), a total of 280 patients were randomized $3: 3: 3: 2$ to subcutaneous injections of lebrikizumab at doses of $125 \mathrm{mg}$ every 4 weeks ( $250 \mathrm{mg}$ loading dose (LD)), $250 \mathrm{mg}$ every 4 weeks (500 mg LD), or $250 \mathrm{mg}$ every 2 weeks ( $500 \mathrm{mg}$ LD at baseline and week 2) or to placebo every 2 weeks or for 16 weeks [55]. At week 16, the lebrikizumab groups showed significant dose-dependent improvements in the percentage change in EASI score (the primary endpoint) vs. placebo: $125 \mathrm{mg}$ every 4 weeks (-62.3\% [37.3\%],
$P=0.02), 250 \mathrm{mg}$ every 4 weeks (-69.2\% [38.3\%], $P=0.002)$, $250 \mathrm{mg}$ every 2 weeks $(-72.1 \%$ [37.2\%], $P<0.001)$, and placebo $(-41.1 \%$ [56.5\%]). Statistically significantly more patients in the $250 \mathrm{mg}$ lebrikizumab-treated group vs. placebo achieved the following secondary endpoints: IGA 0/1 response, EASI 50, EASI 75, and EASI 90, and pruritus NRS score improvement of $\geq 4$ points at week 16 [55].

Most TEAEs were mild to moderate. TEAEs were reported in $46.2 \%$ of placebo patients, $57.5 \%$ of $125 \mathrm{mg}$ Q4W patients, $48.8 \%$ of $250 \mathrm{mg}$ Q4W patients, and $61.3 \%$ of $250 \mathrm{mg}$ Q2W lebrikizumab-treated patients. Among the most frequently reported AEs, upper respiratory tract infections, nasopharyngitis, injection-site pain, and fatigue occurred more frequently with pooled lebrikizumab than with placebo, and headache occurred more frequently with placebo [55].

3.4. Targeted to $I L-31$. Nemolizumab is a humanized monoclonal antibody against the interleukin-31 receptor. In a recently published 16 -week, double-blind, phase 3 clinical trial, a total of 215 Japanese patients ( $\geq 13$ years old) with atopic moderate-to-severe dermatitis were randomized a $2: 1$ ratio to receive subcutaneous nemolizumab $60 \mathrm{mg}$ or placebo every 4 weeks until week 16, with concomitant topical glucocorticoids [56]. At week 16, the mean percent change in the visual analogue scale (VAS) score for pruritus was $-42.8 \%$ in the nemolizumab group and $-21.4 \%$ in the placebo group $(P<0.001)$. The mean percent change in the EASI score (one of the secondary outcomes) was $-45.9 \%$ with nemolizumab and $-33.2 \%$ with placebo [56].

Nemolizumab was generally well tolerated, with an equal TEAE distribution (nemolizumab, 71\%; placebo, 71\%). The most commonly reported adverse event of special interest was worsening atopic dermatitis, occurring in $24 \%$ of the nemolizumab group and $21 \%$ of the placebo group; one patient discontinued nemolizumab as a result. The incidence of injection-related reactions was $8 \%$ in the nemolizumabtreated and $3 \%$ in the placebo-treated patients [56].

In a randomized, double-blind, phase $2 \mathrm{~b}$ study (NCT03100344), a total of 226 adult patients with moderate-to-severe AD were randomized $1: 1: 1: 1$ to receive subcutaneous injections of nemolizumab 10, 30, and $90 \mathrm{mg}$ every 4 weeks or placebo with topical agents [57]. At week 24, among the three nemolizumab-treated groups, the $30 \mathrm{mg}$ dose had the best response rates. The percentage change in EASI score (the primary endpoint) was statistically significant at the $30 \mathrm{mg}$ nemolizumab dose compared with the placebo $(-68.8 \%$ vs. $-52.1 \%, P=0.016)$ and borderline statistically significant at the $10 \mathrm{mg}$ dose $(P=0.051)$. With respect to the secondary endpoints, $36.8 \%$ of subjects in the $30 \mathrm{mg}$ nemolizumab group achieved IGA $0 / 1$ versus $21.1 \%$ in the placebo group $(P=0.06)$. Compared with the placebo, the $30 \mathrm{mg}$ nemolizumab arm achieved the most distinct improvement in peak pruritus NRS (PP-NRS) scores (-68.6\% vs. $-34.3 \%, P<0.0001)$ [57]. The rate of TEAEs was slightly higher in the nemolizumab groups than in the placebo group. Nonskin infections, including nasopharyngitis, upper respiratory tract infections, and gastroenteritis, occurred more frequently with nemolizumab than with placebo [57]. 
TABLE 1: Summary of the new biologics for atopic dermatitis in clinical trials (drugs that did not achieve the primary endpoint*).

\begin{tabular}{|c|c|c|c|c|c|c|}
\hline $\begin{array}{l}\text { Drug } \\
\text { Mechanism } \\
\text { f action } \\
\end{array}$ & $\begin{array}{c}\text { Phase trial } \\
\text { ClinicalTrials.gov } \\
\text { identifier } \\
\end{array}$ & $N$ (ratio), age & Duration & $\begin{array}{c}\text { TCS } \\
(\mathrm{Y} / \mathrm{N} / \mathrm{R})\end{array}$ & Primary endpoint & $\%$ achieving primary endpoint \\
\hline $\begin{array}{l}\text { Fezakinumab } \\
\text { (ILV-094) } \\
\text { Anti-IL-22 }\end{array}$ & $\begin{array}{r}\text { Phase IIa [60] } \\
\text { NCT01941537 }\end{array}$ & $60(2: 1)$, adults & 20 wks & $\mathrm{N}$ & SCORAD & $\begin{array}{c}\text { Entire population: } 300 \mathrm{mg} \text { iv } \\
\text { Q2W: } 13.8 \pm 2.7 \text { vs. placebo: } \\
8.0 \pm 3.1, P=0.134 \\
\text { SCORAD } \geq 50 \text { subgroup: } 300 \mathrm{mg} \text { : } \\
21.6 \pm 3.8 \text { vs. placebo: } 9.6 \pm 4.2 \\
P=0.029\end{array}$ \\
\hline \multirow{2}{*}{$\begin{array}{l}\text { Mepolizumab } \\
\text { (SB240563) } \\
\text { Anti-IL-5 }\end{array}$} & $\begin{array}{l}\text { Phase II [61] } \\
\text { N/A }\end{array}$ & $34(1: 1)$, adults & 20 wks & $\mathrm{N}$ & $\begin{array}{l}\text { IGA } 0 / 1 \text { plus } \geq \\
\text { 2-point (week 16) }\end{array}$ & \multirow{2}{*}{$\begin{array}{l}\text { Failed (100 mg SC Q4W) } \\
\text { Failed: two single doses of } \\
750 \text { mg iv, } P=0.115\end{array}$} \\
\hline & N/A [62] & 43 , adults & 30 days & $\mathrm{R}$ & PGA (14 days) & \\
\hline $\begin{array}{l}\text { Secukinumab } \\
\text { Anti-IL-17A }\end{array}$ & $\begin{array}{l}\text { Phase II [63] } \\
\text { NCT02594098 }\end{array}$ & $41(2: 1)$, adults & 16 wks & $\mathrm{N}$ & $\begin{array}{l}\text { Reduction of } \\
\text { epidermal } \\
\text { thickness } \\
\text { EASI (week 16) }\end{array}$ & $\begin{array}{l}\text { Failed: } 300 \mathrm{mg} \text { qw through week 4, } \\
\text { followed by Q4W to week } 16\end{array}$ \\
\hline $\begin{array}{l}\text { Tezepelumab (AMG } \\
\text { 157/MEDI9929) } \\
\text { Anti-TSLP }\end{array}$ & $\begin{array}{l}\text { Phase IIa [64] } \\
\text { NCT02525094 }\end{array}$ & $\begin{array}{l}113(1: 1) \\
\text { adults }\end{array}$ & 24 wks & $\mathrm{Y}$ & $\begin{array}{l}\text { EASI } 50 \\
\text { (week 12) }\end{array}$ & $\begin{array}{c}\text { Failed: } 280 \mathrm{mg} \text { SC Q2W: } 64.7 \% \text { vs. } \\
\text { placebo: } 48.2 \%, P=0.091\end{array}$ \\
\hline \multirow{2}{*}{$\begin{array}{l}\text { Ustekinumab } \\
\text { Anti-IL12/23p40 }\end{array}$} & $\begin{array}{c}\text { Phase II [65] } \\
\text { NCT01806662 }\end{array}$ & $33(1: 1)$, adults & 32 wks & $\mathrm{Y}$ & $\begin{array}{l}\text { SCORAD } 50 \\
\text { (week 16) }\end{array}$ & Failed ( $45 \mathrm{mg}$ or $90 \mathrm{mg}$ SC 3 doses) \\
\hline & $\begin{array}{l}\text { Phase II [66] } \\
\text { N/A }\end{array}$ & $\begin{array}{l}79(1: 1: 1) \\
\text { (Japanese), } \\
\text { adults }\end{array}$ & 24 wks & $\mathrm{Y}$ & $\begin{array}{l}\text { EASI score } \\
\text { (week 12) }\end{array}$ & Failed (45 mg or $90 \mathrm{mg}$ SC 2 doses) \\
\hline
\end{tabular}

PGA: physician's global assessment. *Fezakinumab (ILV-094): the SCORAD $\geq 50$ subgroup achieved the primary endpoint.

In a phase 2 (part $\mathrm{A}$ ) randomized, double-blind, placebocontrolled study (NCT01986933), a total of 264 adult patients with moderate-to-severe $\mathrm{AD}$ were randomized $1: 1: 1: 1$ to receive subcutaneous nemolizumab (at a dose of $0.1 \mathrm{mg}, 0.5 \mathrm{mg}$, or $2.0 \mathrm{mg}$ per kilogram of body weight) or placebo every 4 weeks or an exploratory dose of $2.0 \mathrm{mg}$ of nemolizumab per kilogram every 8 weeks [58]. At week 12 , the percentage changes in the pruritus visual analogue scale (P-VAS) score (the primary endpoint) were $-43.7 \%$ in the $0.1 \mathrm{mg}$ Q4W group, $-59.8 \%$ in the $0.5 \mathrm{mg} \mathrm{Q} 4 \mathrm{~W}$ group, and $-63.1 \%$ in the $2.0 \mathrm{mg}$ Q $4 \mathrm{~W}$ group versus $-20.9 \%$ in the placebo group ( $P<0.01$ for all comparisons). For the secondary endpoints, changes in the EASI were $-23.0 \%,-42.3 \%$, and $-40.9 \%$, respectively, in the nemolizumab groups versus $-26.6 \%$ in the placebo group. Changes in BSA were $-7.5 \%$, $-20.0 \%$, and $-19.4 \%$, respectively, in the nemolizumab arms versus $-15.7 \%$ in the placebo [58]. The incidence of TEAEs was similar among all groups. The most frequent adverse events included exacerbation of $\mathrm{AD}$, nasopharyngitis, upper respiratory tract infection, peripheral oedema, and increased creatine kinase levels. Exacerbation of $\mathrm{AD}$ and peripheral oedema were more common in the nemolizumab groups than in the placebo group [58].

In a 52-week double-blind extension phase II (part B) trial (NCT01986933), long-term efficacy and safety were assessed in patients who completed part A of the study. Previous placebo patients in part $\mathrm{A}$ were rerandomized $1: 1: 1$ to receive subcutaneous nemolizumab $(0.1,0.5$, or $2.0 \mathrm{mg} / \mathrm{kg}$ $\mathrm{Q} 4 \mathrm{~W})$ in part B [59]. The improvement from baseline on the pruritus visual analogue scale (VAS) score was maintained or increased from weeks 12 to 64 . The greatest improvement was observed in the $0.5 \mathrm{mg} / \mathrm{kg}$ nemolizumab group [59].

At week 64, percentage improvements from baseline in VAS score were $-73.0 \%,-89.6 \%,-74.7 \%$, and $-79.1 \%$ in the $0.1,0.5$, and $2.0 \mathrm{mg} / \mathrm{kg} \mathrm{Q} 4 \mathrm{~W}$ and $2.0 \mathrm{mg} / \mathrm{kg} \mathrm{Q} 8 \mathrm{~W}$ groups, respectively. Changes from baseline in EASI score were $-68.5 \%,-75.8 \%,-78.9 \%$, and $-69.3 \%$ in the $0.1,0.5$, and $2.0 \mathrm{mg} / \mathrm{kg} \mathrm{Q} 4 \mathrm{~W}$ and $2.0 \mathrm{mg} / \mathrm{kg} \mathrm{Q} 8 \mathrm{~W}$ groups, respectively [59]. No new safety concerns were observed after long-term use of nemolizumab. Exacerbation of $\mathrm{AD}(8 \%)$, upper respiratory tract infection (4\%), nasopharyngitis (4\%), peripheral oedema (3\%), increased blood creatine phosphokinase level (3\%), and injection-site reaction (2\%) were the most common treatment-related AEs in the study [59].

Among the abovementioned trials, exacerbation of $\mathrm{AD}$ occurred more frequently in the placebo group in the phase 2B study [57], whereas it occurred more often in the nemolizumab groups in the phase 3 study [56] and phase 2 part A [58]+part B study [59].

3.5. Targeted to $I L-22$. Fezakinumab (ILV-094) is a human monoclonal antibody that directly binds to IL-22 [60]. In a phase 2a randomized, double-blind, placebo-controlled trial (NCT01941537), a total of 60 adult patients were randomized $(2: 1)$ to receive intravenous $300 \mathrm{mg}$ fezakinumab (loading dose of $600 \mathrm{mg}$ at baseline) or placebo every $2 \mathrm{wks}$ for 10 wks [60]. At week 12, the mean decline in SCORAD 


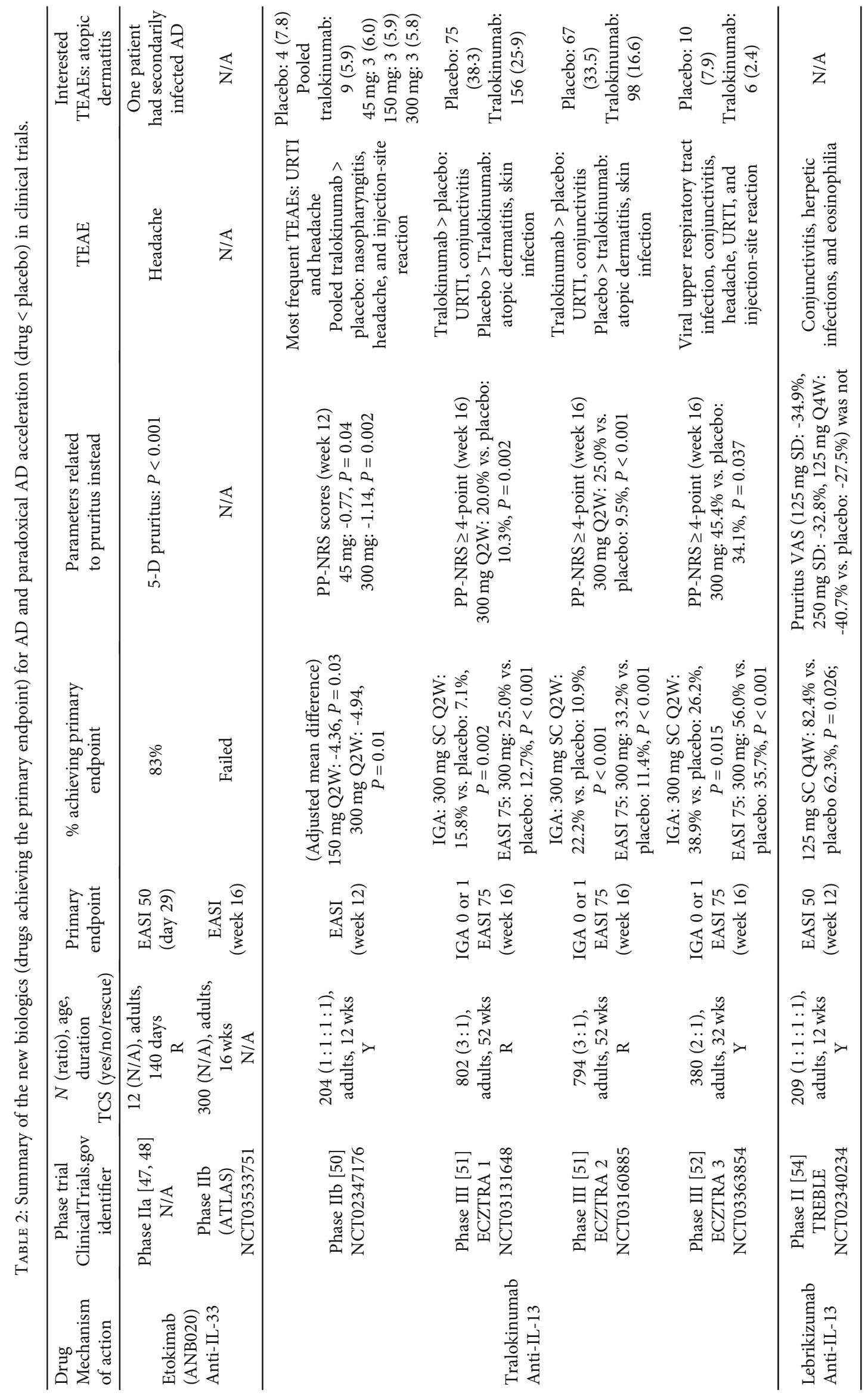




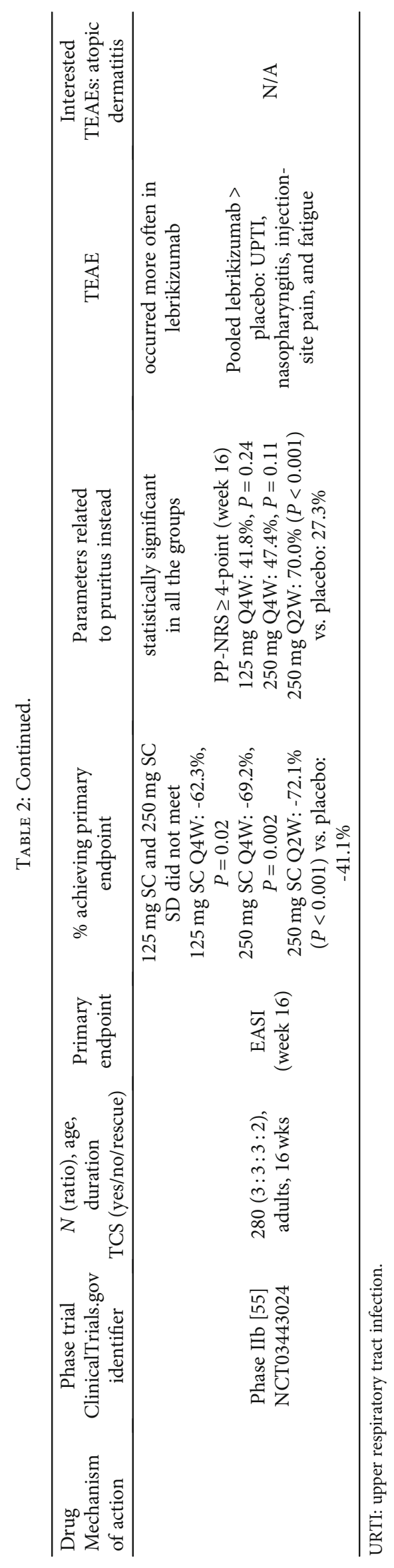




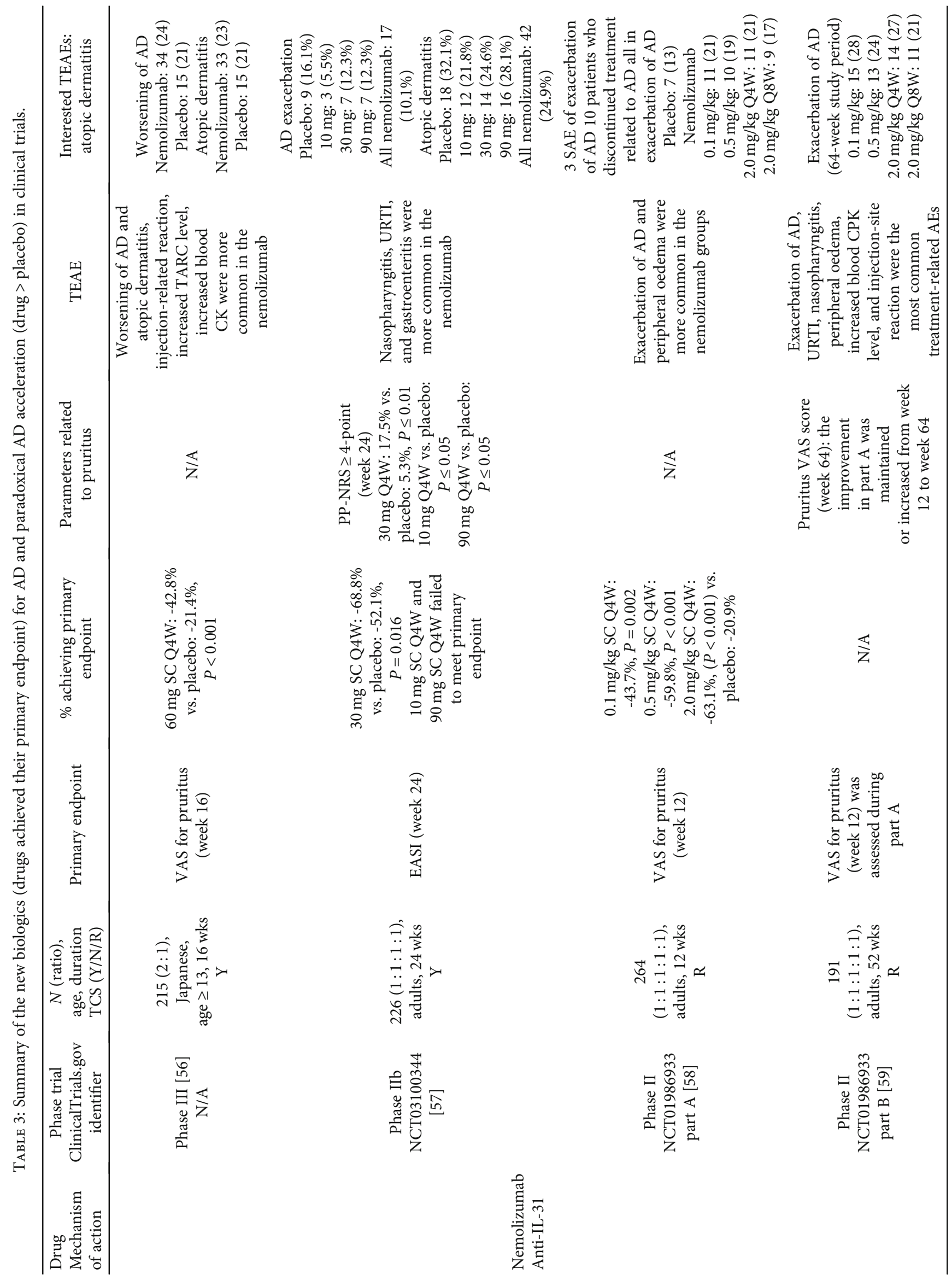




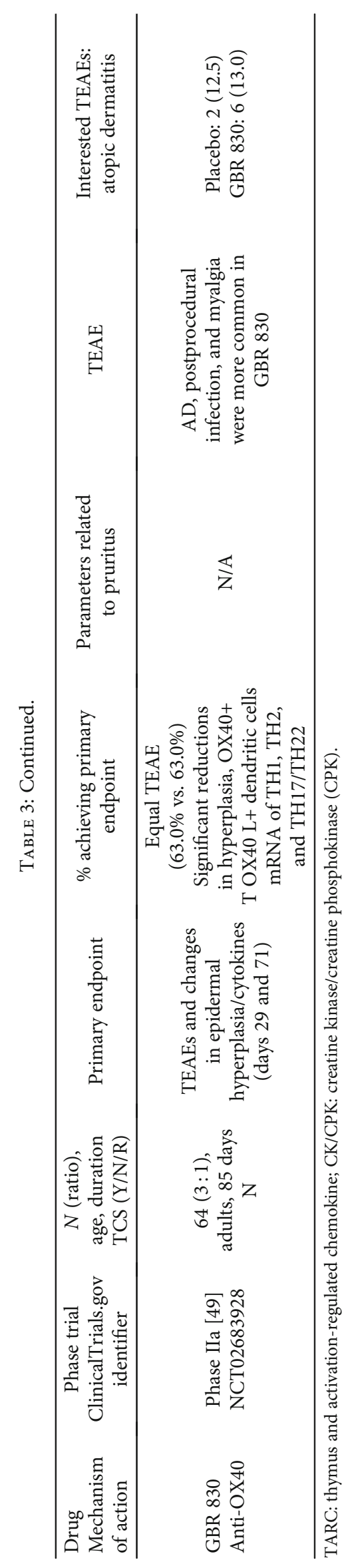


(primary endpoint) for the entire study population was $13.8 \pm 2.7$ (fezakinumab) and $8.0 \pm 3.1$ (placebo), $P=0.134$. In the severe $\mathrm{AD}$ patient subgroup (baseline $\mathrm{SCORAD} \geq 50$ ), the SCORAD reduction was significantly larger in the fezakinumab group than in the placebo group $(21.6 \pm 3.8$ vs. $9.6 \pm$ $4.2, P=0.029)$ and at 20 weeks $(27.4 \pm 3.9$ vs. $11.5 \pm 5.1$, $P=0.010$ ). In nonsevere (moderate) AD patients, none of the efficacy endpoints showed statistically significant differences between the fezakinumab and placebo arms [60]. Adverse events occurred at similar rates between the fezakinumab and placebo groups. Among the most common adverse events that occurred more frequently in the fezakinumab group vs. the placebo group were viral upper respiratory tract infections, occurring in 4 patients receiving fezakinumab [60].

3.6. Targeted to IL-5, IL-17A, TSLP, and IL-12/23p40. Mepolizumab (SB240563): anti-IL-5 [61, 62], secukinumab: anti-IL-17A [63], tezepelumab (AMG 157/MEDI9929): anti-TSLP [64], and ustekinumab: anti-IL12/23p40 [65, 66] all failed to reach their primary endpoint (Table 1).

\section{Discussion}

Among the novel agents discussed here, GBR 830, tralokinumab, lebrikizumab, and nemolizumab have different extents of efficacy, and etokimab showed inconsistent results from different trials. Fezakinumab (targeted to IL22) resulted in significant improvements for only severe patients (SCORAD $\geq 50$ ) [60]. Other drugs, such as tezepelumab (targeted to TSLP), mepolizumab (targeted to IL-5), secukinumab (targeted to IL-17A), and ustekinumab (targeted to IL12/23p40), failed to reach their primary endpoint in clinical trials (Tables 2 and 1).

Among these aforementioned agents, some drugs showed more frequent atopic dermatitis TEAEs than the placebo, including nemolizumab and GBR 830 (Table 3). This atopic dermatitis as a safety outcome was not associated with the efficacy outcome, opening the question of whether some of these cases are paradoxical adverse events. According to the data we showed above and the definition of PAE, some of PAEs may have occurred with these drugs, especially nemolizumab and maybe GBR 830 and the others.

Mechanisms involved in PAEs are complicated. Limited hypotheses have been proposed based on TNF inhibitor investigations. First, an imbalance in the cytokine milieu is advanced during most PAEs [13]. The prototypical example is biological agent-induced psoriasis, due to a TNF- $\alpha /$ type1 IFN cytokine imbalance: TNF-inhibitors (TNFi) block TNF- $\alpha$, which results in uncontrolled activation of plasmacytoid dendritic cells (pDCs), with surplus production of IFN- $\alpha$. IFN- $\alpha$ drives paradoxical skin inflammation [67]. Atopic dermatitis is also an autoimmune disease that involves several cytokines. These cytokines are interwoven in the pathogenesis of $\mathrm{AD}$, and targeting one of these cytokines may have effects on the others. Second, individual genetic susceptibility might play a role [68]. The relationship between $\mathrm{AD}$ and single nucleotide polymorphisms of some genes has been investigated, such as SNPs of the interleu- kin-4/interleukin-13 receptor gene and the $\beta$-defensin 1 gene $[69,70]$. These polymorphisms influence the genes involved in cytokine production, and it is probable that paradoxical reactions occur in patients with an underlying genetic predisposition [14]. This might be one reason why biological agents have been used successfully in some patients with atopic dermatitis, while paradoxically these same types of atopic dermatitis are triggered by the same biological agents. Third, there is a shift in the cutaneous immune response pattern, for example, psoriatic morphology changes (plaque to pustular) [67]. Fourth, a spatial shift of immune cells to the skin [67], for example, a spatial shift of lymphocytes from the gastrointestinal system to the skin, gives rise to the development of psoriasis-like skin inflammation in patients treated for IBD [71]. Fifth, imbalance or dysfunction of regulatory T cells [67], paradoxical cutaneous sarcoidosis and granulomatous disease are prime examples. Drugs targeting TNF result in TNF- $\alpha /$ IL-10 cytokine imbalance and a decrease in TNFR2, followed by dysfunctional Treg increases [72].

Although a few $\mathrm{AD}$ cases of paradoxical reactions to biological therapy have been reported, with their increasing use for $\mathrm{AD}$, an increasing number of reports of paradoxical adverse events of $\mathrm{AD}$ might be seen. Recently, a systematic review of paradoxical eruptions in response to targeted therapies in dermatology was published, and they identified that TNF- $\alpha$ inhibitors resulted in $91.2 \%(1869 / 2049)$ of all cases, followed by IL-17/17R (3.5\%), IL-4R $\alpha$ (2.7\%), IL $12 / 23$ (2.4\%), and IL-23 (0.01\%) inhibitors in 2049 cases of paradoxical reactions. Psoriasiform and eczematous eruptions were the most commonly reported [73].

Biological therapies associated with PAE onset are a challenging issue. Therefore, careful clinical and immunological evaluation should accompany the initiation of biological therapies. In addition, closely monitoring patients receiving biological treatment to detect such reactions is also recommended. These countermeasures will extend our clinical knowledge and shed light on our understanding of the complex immune mechanisms underlying PAEs. The understanding of these new types of adverse reactions will help us to optimize our choices for atopic dermatitis treatment.

\section{Conflicts of Interest}

The authors declare that they have no conflicts of interest.

\section{References}

[1] N. Puar, R. Chovatiya, and A. S. Paller, "New treatments in atopic dermatitis," Annals of Allergy, Asthma \& Immunology, vol. 126, no. 1, pp. 21-31, 2021.

[2] S. Bylund, L. B. von Kobyletzki, M. Svalstedt, and Å. Svensson, "Prevalence and incidence of atopic dermatitis: a systematic review," Acta Dermato Venereologica, vol. 100, no. 12, article adv00160, 2020.

[3] C. J. Arora, F. A. Khattak, M. T. Yousafzai, B. M. Ibitoye, and S. Shumack, "The effectiveness of Janus kinase inhibitors in treating atopic dermatitis: a systematic review and meta-analysis," Dermatologic Therapy, vol. 33, no. 4, article e13685, 2020. 
[4] A. Wollenberg, A. Oranje, M. Deleuran et al., "ETFAD/EADV eczema task force 2015 position paper on diagnosis and treatment of atopic dermatitis in adult and paediatric patients," Journal of the European Academy of Dermatology and Venereology, vol. 30, no. 5, pp. 729-747, 2016.

[5] M. Rademaker, K. Agnew, M. Andrews et al., "Managing atopic dermatitis with systemic therapies in adults and adolescents: an Australian/New Zealand narrative," The Australasian Journal of Dermatology, vol. 61, no. 1, pp. 9-22, 2020.

[6] F. Aubin, F. Carbonnel, and D. Wendling, "The complexity of adverse side-effects to biological agents," Journal of Crohn's \& Colitis, vol. 7, no. 4, pp. 257-262, 2013.

[7] M. Newsom, A. M. Bashyam, E. A. Balogh, S. R. Feldman, and L. C. Strowd, "New and emerging systemic treatments for atopic dermatitis," Drugs, vol. 80, no. 11, pp. 1041-1052, 2020.

[8] 2017 , https://www.fda.gov/news-events/pressannouncements/fda-approves-new-eczema-drug-dupixent.

[9] E. L. Simpson, T. Bieber, E. Guttman-Yassky et al., "Two phase 3 trials of dupilumab versus placebo in atopic dermatitis," The New England Journal of Medicine, vol. 375, no. 24, pp. 23352348, 2016.

[10] M. Worm, E. L. Simpson, D. Thaçi et al., "Efficacy and safety of multiple dupilumab dose regimens after initial successful treatment in patients with atopic dermatitis: a randomized clinical trial," JAMA Dermatology, vol. 156, no. 2, pp. 131$143,2020$.

[11] M. de Bruin-Weller, D. Thaçi, C. H. Smith et al., "Dupilumab with concomitant topical corticosteroid treatment in adults with atopic dermatitis with an inadequate response or intolerance to ciclosporin A or when this treatment is medically inadvisable: a placebo-controlled, randomized phase III clinical trial (LIBERTY AD CAFÉ)," The British Journal of Dermatology, vol. 178, no. 5, pp. 1083-1101, 2018.

[12] L. Puig, "Paradoxical reactions: anti-tumor necrosis factor alpha agents, ustekinumab, secukinumab, ixekizumab, and others," in Adverse reactions to biologics, vol. 53, pp. 49-63, Karger Publishers, 2018.

[13] É. Toussirot and F. Aubin, "Paradoxical reactions under TNF$\alpha$ blocking agents and other biological agents given for chronic immune-mediated diseases: an analytical and comprehensive overview," RMD Open, vol. 2, no. 2, article e000239, 2016.

[14] M. Munera-Campos, F. Ballesca, and J. M. Carrascosa, "Reacciones paradojicas de los tratamientos biologicos utilizados en psoriasis: revision de la literatura," Actas Dermo-Sifiliográficas (English Edition), vol. 109, no. 9, pp. 791-800, 2018.

[15] F. J. Navarro-Trivino, R. Sanchez-Parera, and R. Ruiz-Villaverde, "Secukinumab-induced paradoxical hidradenitis suppurativa," Dermatologic Therapy, vol. 33, no. 1, article e13150, 2020.

[16] L. Jaulent, D. Staumont-Sallé, M. Tauber et al., "De novo psoriasis in atopic dermatitis patients treated with dupilumab: a retrospective cohort," Journal of the European Academy of Dermatology and Venereology, vol. 35, no. 4, pp. e296-e297, 2021.

[17] A. Varma and J. Levitt, "Dupilumab-induced phenotype switching from atopic dermatitis to psoriasis," JAAD Case Reports, vol. 6, no. 3, pp. 217-218, 2020.

[18] M. Napolitano, M. Scalvenzi, G. Fabbrocini, E. Cinelli, and C. Patruno, "Occurrence of psoriasiform eruption during dupilumab therapy for adult atopic dermatitis: a case series," Dermatologic Therapy, vol. 32, no. 6, article e13142, 2019.
[19] I. D'Ambra, G. Babino, E. Fulgione et al., "Psoriasis onset under dupilumab treatment in two patients affected by atopic dermatitis and one patient affected by alopecia areata: clinical and dermoscopic patterns," Dermatologic Therapy, vol. 33, no. 6, article e14169, 2020.

[20] H. S. Kim and J. Yeung, "Psoriasis appearing after dupilumab therapy in atopic dermatitis: a case report," SAGE Open Medical Case Reports, vol. 8, 2020.

[21] M. Stout, J. Guitart, T. Tan, and J. I. Silverberg, "Psoriasis-like dermatitis developing in a patient with atopic dermatitis treated with dupilumab," Dermatitis, vol. 30, no. 6, pp. 376378, 2019.

[22] N. Gori, G. Caldarola, F. Pirro, C. De Simone, and K. Peris, "A case of guttate psoriasis during treatment with dupilumab," Dermatologic Therapy, vol. 32, no. 5, article e12998, 2019.

[23] S. Ferrucci, S. Tavecchio, E. Berti, and L. Angileri, “Acute onset of psoriasis in a patient with atopic dermatitis treated with dupilumab," Clinical and Experimental Dermatology, vol. 45, no. 5 , pp. 625-626, 2020.

[24] R. A. Waldman, M. E. DeWane, B. Sloan, and J. M. Grant-Kels, "Characterizing dupilumab facial redness: a multi-institution retrospective medical record review," Journal of the American Academy of Dermatology, vol. 82, no. 1, pp. 230-232, 2020.

[25] S. Muzumdar, M. Zubkov, R. Waldman, M. E. DeWane, R. Wu, and J. M. Grant-Kels, "Characterizing dupilumab facial redness in children and adolescents: a single- institution retrospective chart review," Journal of the American Academy of Dermatology, vol. 83, no. 5, pp. 1520-1521, 2020.

[26] L. E. M. Wijs, N. T. Nguyen, A. C. M. Kunkeler, T. Nijsten, J. Damman, and D. J. Hijnen, "Clinical and histopathological characterization of paradoxical head and neck erythema in patients with atopic dermatitis treated with dupilumab: a case series," The British Journal of Dermatology, vol. 183, no. 4, pp. 745-749, 2020.

[27] R. Nixon, R. Despiney, and P. Pfeffer, "Case of paradoxical adverse response to mepolizumab with mepolizumabinduced alopecia in severe eosinophilic asthma," BMJ Case Reports, vol. 13, no. 2, article e233161, 2020.

[28] Y. Ishiuji, Y. Umezawa, A. Asahina et al., "Exacerbation of atopic dermatitis symptoms by ustekinumab in psoriatic patients with elevated serum immunoglobulin E levels: report of two cases," The Journal of Dermatology, vol. 45, no. 6, pp. 732-734, 2018.

[29] E. Guttman-Yassky, A. Waldman, J. Ahluwalia, P. Y. Ong, and L. Eichenfield, "Atopic dermatitis: pathogenesis," Seminars in Cutaneous Medicine and Surgery, vol. 36, no. 3, pp. 100-103, 2017.

[30] D. J. Margolis, A. J. Apter, J. Gupta et al., "The persistence of atopic dermatitis and filaggrin (FLG) mutations in a US longitudinal cohort," The Journal of Allergy and Clinical Immunology, vol. 130, no. 4, pp. 912-917, 2012.

[31] M. Furue, D. Ulzii, Y. H. Vu, G. Tsuji, M. Kido-Nakahara, and T. Nakahara, "Pathogenesis of atopic dermatitis: current paradigm," Iranian Journal of Immunology, vol. 16, no. 2, pp. 97107, 2019.

[32] E. Volpe, L. Pattarini, C. Martinez-Cingolani et al., "Thymic stromal lymphopoietin links keratinocytes and dendritic cellderived IL-23 in patients with psoriasis," The Journal of Allergy and Clinical Immunology, vol. 134, no. 2, pp. 373-381.e4, 2014.

[33] M. Munera-Campos and J. M. Carrascosa, "Innovacion en dermatitis atopica: de la patogenia a la terapeutica," Actas 
Dermo-Sifiliográficas (English Edition), vol. 111, no. 3, pp. 205-221, 2020.

[34] N. Murakami-Satsutani, T. Ito, T. Nakanishi et al., "IL-33 promotes the induction and maintenance of Th2 immune responses by enhancing the function of OX40 ligand," Allergology International, vol. 63, no. 3, pp. 443-455, 2014.

[35] Y. Renert-Yuval and E. Guttman-Yassky, "New treatments for atopic dermatitis targeting beyond IL-4/IL-13 cytokines," Annals of Allergy, Asthma \& Immunology, vol. 124, no. 1, pp. 28-35, 2020.

[36] Y. Mansouri and E. Guttman-Yassky, "Immune pathways in atopic dermatitis, and definition of biomarkers through broad and targeted therapeutics," Journal of Clinical Medicine, vol. 4, no. 5, pp. 858-873, 2015.

[37] J. Wu and E. Guttman-Yassky, "Efficacy of biologics in atopic dermatitis," Expert Opinion on Biological Therapy, vol. 20, no. 5, pp. 525-538, 2020.

[38] E. Guttman-Yassky, R. Bissonnette, B. Ungar et al., "Dupilumab progressively improves systemic and cutaneous abnormalities in patients with atopic dermatitis," The Journal of Allergy and Clinical Immunology, vol. 143, no. 1, pp. 155$172,2019$.

[39] E. Sonkoly, A. Muller, A. I. Lauerma et al., "IL-31: a new link between T cells and pruritus in atopic skin inflammation," The Journal of Allergy and Clinical Immunology, vol. 117, no. 2, pp. 411-417, 2006.

[40] A.-x. Wang and N. X. Landén, "New insights into T cells and their signature cytokines in atopic dermatitis," IUBMB Life, vol. 67 , no. 8, pp. 601-610, 2015.

[41] M. Suárez-Fariñas, N. Dhingra, J. Gittler et al., "Intrinsic atopic dermatitis shows similar $\mathrm{T}_{\mathrm{H}} 2$ and higher $\mathrm{T}_{\mathrm{H}} 17$ immune activation compared with extrinsic atopic dermatitis," The Journal of Allergy and Clinical Immunology, vol. 132, no. 2, pp. 361-370, 2013.

[42] S. Noda, M. Suárez-Fariñas, B. Ungar et al., "The Asian atopic dermatitis phenotype combines features of atopic dermatitis and psoriasis with increased $\mathrm{T}_{\mathrm{H}} 17$ polarization," The Journal of Allergy and Clinical Immunology, vol. 136, no. 5, pp. 1254-1264, 2015.

[43] T. Czarnowicki, H. He, J. G. Krueger, and E. Guttman-Yassky, "Atopic dermatitis endotypes and implications for targeted therapeutics," The Journal of Allergy and Clinical Immunology, vol. 143, no. 1, pp. 1-11, 2019.

[44] K. Kikly, L. Liu, S. Na, and J. D. Sedgwick, “The IL-23/Th 17 axis: therapeutic targets for autoimmune inflammation," Current Opinion in Immunology, vol. 18, no. 6, pp. 670675, 2006.

[45] A. D. Cesare, P. D. Meglio, and F. O. Nestle, "A role for Th17 cells in the immunopathogenesis of atopic dermatitis?," The Journal of Investigative Dermatology, vol. 128, no. 11, pp. 2569-2571, 2008.

[46] M. W. L. Teng, E. P. Bowman, J. J. McElwee et al., "IL-12 and IL-23 cytokines: from discovery to targeted therapies for immune-mediated inflammatory diseases," Nature Medicine, vol. 21, no. 7, pp. 719-729, 2015.

[47] Y.-L. Chen, D. Gutowska-Owsiak, C. S. Hardman et al., "Proof-of-concept clinical trial of etokimab shows a key role for IL-33 in atopic dermatitis pathogenesis," Science Translational Medicine, vol. 11, no. 515, article eaax2945, 2019.

[48] G. Ogg, "Proof-of-concept phase 2a clinical trial of ANB020 (anti-IL-33 antibody) in the treatment of moderate-to-severe atopic dermatitis," in AAD annual meeting, San Diego, CA, USA, 2018.

[49] E. Guttman-Yassky, A. B. Pavel, L. Zhou et al., "GBR 830, an anti-OX40, improves skin gene signatures and clinical scores in patients with atopic dermatitis," Journal of Allergy and Clinical Immunology, vol. 144, no. 2, pp. 482-493.e7, 2019.

[50] A. Wollenberg, M. D. Howell, E. Guttman-Yassky et al., "Treatment of atopic dermatitis with tralokinumab, an antiIL-13 mAb," The Journal of Allergy and Clinical Immunology, vol. 143, no. 1, pp. 135-141, 2019.

[51] A. Wollenberg, A. Blauvelt, E. Guttman-Yassky et al., “Tralokinumab for moderate-to-severe atopic dermatitis: results from two 52-week, randomized, double-blind, multicentre, placebo-controlled phase III trials (ECZTRA 1 and ECZTRA 2)," The British Journal of Dermatology, vol. 184, no. 3, pp. 437-449, 2021.

[52] J. I. Silverberg, D. Toth, T. Bieber et al., "Tralokinumab plus topical corticosteroids for the treatment of moderate-tosevere atopic dermatitis: results from the double-blind, randomized, multicentre, placebo-controlled phase III ECZTRA 3 trial," The British Journal of Dermatology, vol. 184, no. 3, pp. 450-463, 2021.

[53] M. Ultsch, J. Bevers, G. Nakamura et al., "Structural basis of signaling blockade by anti-IL-13 antibody lebrikizumab," Journal of Molecular Biology, vol. 425, no. 8, pp. 1330-1339, 2013.

[54] E. L. Simpson, C. Flohr, L. F. Eichenfield et al., "Efficacy and safety of lebrikizumab (an anti-IL-13 monoclonal antibody) in adults with moderate-to-severe atopic dermatitis inadequately controlled by topical corticosteroids: a randomized, placebo-controlled phase II trial (TREBLE)," Journal of the American Academy of Dermatology, vol. 78, no. 5, pp. 863871.e11, 2018.

[55] E. Guttman-Yassky, A. Blauvelt, L. F. Eichenfield et al., "Efficacy and safety of lebrikizumab, a high-affinity interleukin 13 inhibitor, in adults with moderate to severe atopic dermatitis: a phase $2 \mathrm{~b}$ randomized clinical trial," JAMA Dermatology, vol. 156, no. 4, pp. 411-420, 2020.

[56] K. Kabashima, T. Matsumura, H. Komazaki, and M. Kawashima, "Trial of nemolizumab and topical agents for atopic dermatitis with pruritus," The New England Journal of Medicine, vol. 383, no. 2, pp. 141-150, 2020.

[57] J. I. Silverberg, A. Pinter, G. Pulka et al., "Phase 2B randomized study of nemolizumab in adults with moderate-to-severe atopic dermatitis and severe pruritus," The Journal of Allergy and Clinical Immunology, vol. 145, no. 1, pp. 173-182, 2020.

[58] T. Ruzicka, J. M. Hanifin, M. Furue et al., "Anti-interleukin-31 receptor a antibody for atopic dermatitis," The New England Journal of Medicine, vol. 376, no. 9, pp. 826-835, 2017.

[59] K. Kabashima, M. Furue, J. M. Hanifin et al., "Nemolizumab in patients with moderate-to-severe atopic dermatitis: randomized, phase II, long-term extension study," Journal of Allergy and Clinical Immunology, vol. 142, no. 4, pp. 1121-1130.e7, 2018.

[60] E. Guttman-Yassky, P. M. Brunner, A. U. Neumann et al., "Efficacy and safety of fezakinumab (an IL-22 monoclonal antibody) in adults with moderate-to-severe atopic dermatitis inadequately controlled by conventional treatments: a randomized, double-blind, phase 2a trial," Journal of the American Academy of Dermatology, vol. 78, no. 5, pp. 872-881.e6, 2018.

[61] E. G. Kang, P. K. Narayana, I. J. Pouliquen, M. C. Lopez, M. C. Ferreira-Cornwell, and J. A. Getsy, "Efficacy and safety of mepolizumab administered subcutaneously for moderate to 
severe atopic dermatitis," Allergy, vol. 75, no. 4, pp. 950-953, 2020.

[62] J. M. Oldhoff, U. Darsow, T. Werfel et al., “Anti-IL-5 recombinant humanized monoclonal antibody (mepolizumab) for the treatment of atopic dermatitis," Allergy, vol. 60, no. 5, pp. 693696, 2005.

[63] B. Ungar, A. B. Pavel, R. Li et al., "Phase 2 randomized, doubleblind study of IL-17 targeting with secukinumab in atopic dermatitis," The Journal of Allergy and Clinical Immunology, vol. 147, no. 1, pp. 394-397, 2021.

[64] E. L. Simpson, J. R. Parnes, D. She et al., “Tezepelumab, an anti-thymic stromal lymphopoietin monoclonal antibody, in the treatment of moderate to severe atopic dermatitis: a randomized phase 2a clinical trial," Journal of the American Academy of Dermatology, vol. 80, no. 4, pp. 1013-1021, 2019.

[65] S. Khattri, P. M. Brunner, S. Garcet et al., "Efficacy and safety of ustekinumab treatment in adults with moderate-to-severe atopic dermatitis," Experimental Dermatology, vol. 26, no. 1, pp. 28-35, 2017.

[66] H. Saeki, K. Kabashima, Y. Tokura et al., "Efficacy and safety of ustekinumab in Japanese patients with severe atopic dermatitis: a randomized, double-blind, placebo-controlled, phase II study," The British Journal of Dermatology, vol. 177, no. 2, pp. 419-427, 2017.

[67] S. Garcovich, C. De Simone, G. Genovese, E. Berti, M. Cugno, and A. V. Marzano, "Paradoxical skin reactions to biologics in patients with rheumatologic disorders," Frontiers in Pharmacology, vol. 10, p. 282, 2019.

[68] M. Ramos-Casals, C. D.-L. Roberto-Perez-Alvarez, M.J. Cuadrado, and M. A. Khamashta, "Autoimmune diseases induced by biological agents: a double-edged sword?," Autoimmunity Reviews, vol. 9, no. 3, pp. 188-193, 2010.

[69] N. Novak, S. Kraft, E. Geiger et al., "Dichotomic nature of atopic dermatitis reflected by combined analysis of monocyte immunophenotyping and single nucleotide polymorphisms of the interleukin-4/interleukin-13 receptor gene: the dichotomy of extrinsic and intrinsic atopic dermatitis," Journal of Investigative Dermatology, vol. 119, no. 4, pp. 870-875, 2002.

[70] E. P.-M. de Oca, A. García-Vargas, R. Lozano-Inocencio et al., "Association of beta-defensin 1 single nucleotide polymorphisms with atopic dermatitis," International Archives of Allergy and Immunology, vol. 142, no. 3, pp. 211-218, 2007.

[71] K. Eyerich and S. Eyerich, "Immune response patterns in noncommunicable inflammatory skin diseases," Journal of the European Academy of Dermatology and Venereology, vol. 32, no. 5, pp. 692-703, 2018.

[72] K. T. Amber, R. Bloom, U. Mrowietz, and M. Hertl, “TNF- $\alpha$ : a treatment target or cause of sarcoidosis?," Journal of the European Academy of Dermatology and Venereology, vol. 29, no. 11, pp. 2104-2111, 2015.

[73] M. J. Murphy, J. M. Cohen, M. D. Vesely, and W. Damsky, "Paradoxical eruptions to targeted therapies in dermatology: a systematic review and analysis," Journal of the American Academy of Dermatology, vol. S0190-9622, no. 20, pp. 33154-33156, 2020. 